\title{
One-loop conformal anomaly in an implicit momentum space regularization framework
}

\author{
A. R. Vieira ${ }^{1, a}$, J. C. C. Felipe ${ }^{2}$, G. Gazzola ${ }^{1}$, Marcos Sampaio ${ }^{1,3}$ \\ ${ }_{1}^{1}$ Departamento de Física, ICEX, Universidade Federal de Minas Gerais, P.O. Box 702, Belo Horizonte, MG 30.161-970, Brazil \\ ${ }^{2}$ Departamento de Física, Universidade Federal de Lavras, P.O. Box 3037, Lavras, MG 37.200-000, Brazil \\ ${ }^{3}$ Centre for Particle Theory, Department of Mathematical Sciences, Durham University, Science Laboratories South Rd., Durham DH1 3LE, UK
}

Received: 20 May 2015 / Accepted: 8 July 2015 / Published online: 18 July 2015

(C) The Author(s) 2015. This article is published with open access at Springerlink.com

\begin{abstract}
In this paper we consider matter fields in a gravitational background in order to compute the breaking of the conformal current at one-loop order. Standard perturbative calculations of conformal symmetry breaking expressed by the non-zero trace of the energy-momentum tensor have shown that some violating terms are regularization dependent, which may suggest the existence of spurious breaking terms in the anomaly. Therefore, we perform the calculation in a momentum space regularization framework in which regularization dependent terms are judiciously parametrized. We compare our results with those obtained in the literature and conclude that there is an unavoidable arbitrariness in the anomalous term $\square R$.
\end{abstract}

\section{Introduction}

Conformal invariance (CI) imposes strong constraints on the correlation functions leading to exact results mainly in two dimensions. On the other hand most renormalizable theories possessing conformal invariance at the classical level exhibit the trace anomaly once quantum corrections are taken into account. Of course this is most welcome in particle physics because conformal symmetry breaking must come into play to properly describe the real world. Then, in the high energy limit, CI may be recovered appearing as UV and/or IR limits [1]. Furthermore, CI is an important concept in holographic theories based on the $\mathrm{AdS}_{n+1} / \mathrm{CFT}_{n}$ duality which relates strongly coupled four dimensional gauge theory to gravitational theory in five dimensional AdS space-time, for instance. It is also important in super-symmetric gauge theories, e.g. (conformal invariant) $\mathcal{N}=4$ super Yang-Mills. For applications of the AdS/CFT conjecture [2] in many branches of physics see [3].

\footnotetext{
a e-mail: arvieira@ fisica.ufmg.br
}

Anomalies occur when a symmetry presented at a classical level is broken upon quantization. In perturbation theory, during the process of regularization/renormalization, counter-terms are generated and may violate the symmetry that was present at the classical level. The presence of anomaly depends on the fact that it is not possible to find a regulator that preserves all the symmetries of the classical action. Well-known examples of anomalies are the (AVV) chiral anomaly $[4,5]$ when gauge fields coupled to conserved currents give rise to non-conserved axial current, and the trace anomaly of a scalar field conformally coupled to a classical gravitational background $[6,7]$.

Finite and undetermined local terms appear as differences between loop integrals with the same degree of divergence in Feynman diagram calculations [8]. Such indeterminacies are regularization dependent and are at the heart of symmetry breakings by regularizations. A reasonable strategy would be to leave them arbitrary till the end of the calculation to be fixed on symmetry or physical grounds. Anomalies, such as the AVV chiral anomaly, appear in this approach when the ambiguities proved themselves insufficient to preserve the full set of symmetry identities valid at classical level.

Attributing spurious values to such indeterminacies can break gauge invariance or super-symmetry [9]. In the latter reference it was shown that undetermined local terms can be cast as surface terms at any loop order. Moreover, it was argued that momentum routing invariance (MRI) is a necessary and sufficient condition to preserve (abelian) gauge symmetry at arbitrary loop order. This condition is automatically fulfilled by dimensional regularization [10]. The strategy of identifying ambiguous regularization dependent surface terms in perturbation theory to arbitrary loop order is better understood and accomplished within implicit regularization (IR) [11], which is discussed in more detail in Sect. 3. 
IR is a momentum space setting to perform Feynman diagram calculations in a regularization independent fashion. Consequently IR turns out particularly adequate to unravel anomalies within perturbation theory. In IR, the Lagrangian of the underlying quantum field theory is not modified because neither an explicit regulator is introduced nor the dimensionality of the space time needs to be moved away from its physical dimension. In particular, IR allows for a democratic display of the anomaly between the Ward identities which ultimately should be fixed on physical grounds. For example, in [12] was studied Weyl fermions on a classical gravitational background in two dimensions and shown that, assuming Lorentz symmetry, the Weyl and EinsteinWard identities reduce to a set of algebraic equations for the arbitrary parameters which place the Ward identities on equal footing, just as in the AVV triangle anomaly [9].

In this contribution we revisit an old controversy related to breaking of the conformal current at one-loop order when matter fields lie on a gravitational background. Some of the terms of this anomaly are ambiguous and regularization dependent $[13,14]$. Therefore, we investigate if this ambiguity appears as surface terms which sometimes may be fixed on symmetry grounds. Moreover, we believe that performing this calculation in four dimensions and without introducing an explicit regulator is worthwhile, since we will not get spurious terms that may contaminate the anomaly.

This work is organized as follows: in Sect. 2 we review some aspects about conformal anomaly; in Sect. 3 we outline the implicit regularization scheme to establish our notation; in Sect. 4 we derive the $a^{\prime}$ coefficient using the one-loop correction to the graviton propagator; in Sect. 5 we perform the one-loop renormalization of the quantum effective action; we present how the anomaly is affected by the surface terms in Sect. 6 and we draw concluding remarks in Sect. 7.

\section{Aperçu on conformal anomaly}

In order to present the state of the art let us establish some notation. A theory is conformal invariant if it does not change under the field transformation

$\Psi^{\prime}(x)=e^{d \sigma(x)} \Psi(x)$,

where $\Psi$ stands for scalar, vector, spinor or the metric ( $\Psi=$ $\phi, A_{\mu}, \psi$ or $g_{\mu \nu}$, respectively), $\sigma$ is an arbitrary scalar field and $d$ is the corresponding conformal weight for the scalar, vector or spinor fields ( $d=-1,0$, and $-\frac{3}{2}$, respectively) and it is equal to 2 for the metric.

The corresponding conserved current associated with the transformation (1) is the conformal current also known as the trace of the energy-momentum tensor. In classical field theory, this current is conserved in the massless limit. Quan- tum corrections usually break conformal invariance in the semi-classical approach of gravity (see [13] for a review). Pioneering works about this anomaly have derived one-loop corrections to the graviton propagator due to vector [6] and spinor [15] couplings. They found that, although the diffeomorphism was preserved, the trace of the energy-momentum tensor was no longer zero at the quantum level [16], since it received finite corrections. Like other anomalies this breaking poses a renormalizability issue [17].

At first, this symmetry breaking was thought of as being spurious [18-20], that is to say, an artifact of the regularization method, motivating the seek for a regularization scheme which preserves both CI and diffeomorphism [21-24]. Afterwards, the trace of energy-momentum tensor was computed in several frameworks. In [16] it was calculated diagrammatically using dimensional regularization [10,25]. Moreover, it was shown that the anomaly also arises in $\zeta$-function regularization [26], point-splitting regularization [27,28] and in the context of Schwinger-DeWitt method [29,30]. A derivation based on the AdS/CFT correspondence can be found in [31]. Besides, this anomaly has already been classified in a regularization independent way using the algebraic approach [32]. However, the explicit diagrammatic computation reveals that some of the terms which quantum mechanically break conformal invariance are regularization dependent. For a review of conformal anomaly and its universalities and ambiguities in different regularization schemes, see [14].

It is noteworthy that the anomalous trace of the energymomentum tensor has physical consequences: it determines the energy-momentum tensor for a black hole in two dimensions [34] and the classification of the vacuum quantum states in four dimensions $[35,36]$. This anomaly also gives rise to the stability condition in the modified Starobinski inflationary model $[37,38]$. Besides, the anomaly induced action has applications in black hole evaporation [39], annihilation of an AdS universe [40] and creation of a de Sitter wall universe [41].

The trace anomaly has a general form given by

$T=\left\langle T_{\mu}^{\mu}\right\rangle=a C^{2}+c E+a^{\prime} \square R$,

where $C^{2}=R_{\mu \nu \alpha \beta}^{2}-2 R_{\alpha \beta}^{2}+\frac{1}{3} R^{2}$ is the square of the Weyl tensor, $E=R_{\mu \nu \alpha \beta}^{2}-4 R_{\alpha \beta}^{2}+R^{2}$ is the Gauss-Bonnet topological invariant, $R$ is the Ricci scalar and $a, c$, and $a^{\prime}$ are related with $\beta$-functions [14]

$$
\begin{aligned}
& \beta_{1}=\frac{1}{(4 \pi)^{2}}\left(\frac{1}{120} N_{s}+\frac{1}{20} N_{f}+\frac{1}{10} N_{v}\right), \\
& \beta_{2}=\frac{1}{(4 \pi)^{2}}\left(\frac{1}{360} N_{s}+\frac{11}{360} N_{f}+\frac{31}{180} N_{v}\right), \\
& \beta_{3}=\frac{1}{(4 \pi)^{2}}\left(\frac{1}{180} N_{s}+\frac{1}{30} N_{f}-\frac{1}{10} N_{v}\right) .
\end{aligned}
$$


The usual results in the literature are $a=\beta_{1}$ and $c=\beta_{2}$. However, there is a disagreement in the coefficient $a^{\prime}$. While some regularization schemes predict $a^{\prime}=\beta_{3}$, dimensional regularization yields $a^{\prime}=\frac{2}{3} \beta_{1}$ [16]. Furthermore, $a^{\prime}$ vanishes in the derivation based on the AdS/CFT [31] correspondence and it is ambiguous in Pauli-Villars regularization $[14,42,43]$.

Afterwards, it was shown that dimensional regularization actually also furnishes an ambiguous result [14].

We shall compute the trace anomaly in an implicit momentum space regularization framework, paying particular attention to regularization dependent quantities [8,9]. We perform the one-loop correction to the graviton propagator due to couplings with scalar, fermion, and vector fields. We then relate that correction for the two-point function with $\left\langle T_{\mu}^{\mu}\right\rangle$. For this purpose we employ implicit regularization [11] in which divergences are expressed order by order in perturbation theory as loop integrals in consonance with BPHZ theorem [44] whereas undetermined regularization dependent local terms are expressed by surface terms. Thus we derive the $a^{\prime}$ coefficient and then compare our result with those of the literature.

\section{Implicit regularization}

We apply the implicit regularization framework [11] to treat the integrals which appear in the amplitudes of Sect. 4. Let us make a brief review of the method. In this scheme, we assume the existence of an implicit regulator $\Lambda$ just to justify algebraic operations within the integrands. We then use the following identity to separate UV divergent basic integrals from the finite part:

$$
\begin{aligned}
\int_{k} \frac{1}{(k+p)^{2}-m^{2}}= & \int_{k} \frac{1}{k^{2}-m^{2}} \\
& -\int_{k} \frac{\left(p^{2}+2 p \cdot k\right)}{\left(k^{2}-m^{2}\right)\left[(k+p)^{2}-m^{2}\right]}
\end{aligned}
$$

where $\int_{k} \equiv \int^{\Lambda} \frac{d^{4} k}{(2 \pi)^{4}}$, to separate basic divergent integrals (BDI's) from the finite part. These BDI's are defined as follows:

$$
\begin{aligned}
& I_{\log }^{\mu_{1} \cdots \mu_{2 n}}\left(m^{2}\right) \equiv \int_{k} \frac{k^{\mu_{1}} \cdots k^{\mu_{2 n}}}{\left(k^{2}-m^{2}\right)^{2+n}}, \\
& I_{\text {quad }}^{\mu_{1} \cdots \mu_{2 n}}\left(m^{2}\right) \equiv \int_{k} \frac{k^{\mu_{1}} \cdots k^{\mu_{2 n}}}{\left(k^{2}-m^{2}\right)^{1+n}}
\end{aligned}
$$

and

$$
I_{\text {quart }}^{\mu_{1} \cdots \mu_{2 n}}\left(m^{2}\right) \equiv \int_{k} \frac{k^{\mu_{1}} \cdots k^{\mu_{2 n}}}{\left(k^{2}-m^{2}\right)^{n}}
$$

The basic divergences with Lorentz indices can be combined as differences between integrals with the same superficial degree of divergence, according to the equations below, which define surface terms ${ }^{1}$ :

$$
\begin{aligned}
& \Upsilon_{2 w}^{\mu \nu}=\eta^{\mu \nu} I_{2 w}\left(m^{2}\right)-2(2-w) I_{2 w}^{\mu \nu}\left(m^{2}\right) \equiv v_{2 w} \eta^{\mu \nu}, \\
& \Xi_{2 w}^{\mu \nu \alpha \beta}=\eta^{\{\mu \nu} \eta^{\alpha \beta\}} I_{2 w}\left(m^{2}\right) \\
& \quad-4(3-w)(2-w) I_{2 w}^{\mu \nu \alpha \beta}\left(m^{2}\right) \equiv \xi_{2 w} \eta^{\{\mu \nu} \eta^{\alpha \beta\}}, \\
& \Sigma_{2 w}^{\mu \nu \alpha \beta \gamma \delta}=\eta^{\{\mu \nu} \eta^{\alpha \beta} \eta^{\gamma \delta\}} I_{2 w}\left(m^{2}\right) \\
& \quad-8(4-w)(3-w)(2-w) I_{2 w}^{\mu \nu \alpha \beta \gamma \delta}\left(m^{2}\right) \\
& \equiv \sigma_{2 w} \eta^{\{\mu \nu} \eta^{\alpha \beta} \eta^{\gamma \delta\}} \\
& \Omega_{2 w}^{\mu \nu \alpha \beta \gamma \delta \epsilon \zeta}=\eta^{\{\mu \nu} \eta^{\alpha \beta} \eta^{\gamma \delta} \eta^{\epsilon \zeta\}} I_{2 w}\left(m^{2}\right) \\
& \quad-16(5-w)(4-w)(3-w)(2-w) \\
& \quad \times I_{2 w}^{\mu \nu \alpha \beta \gamma \delta \epsilon \zeta}\left(m^{2}\right) \\
& \equiv \omega_{2 w} \eta^{\{\mu \nu} \eta^{\alpha \beta} \eta^{\gamma \delta} \eta^{\epsilon \zeta\}} .
\end{aligned}
$$

In the expressions above, $2 w$ is the degree of divergence of the integrals and, for the sake of brevity, we substitute the subscripts $\log$, quad, and quart by 0,2 , and 4 , respectively. Surface terms can be conveniently written as integrals of total derivatives, namely

$v_{2 w} \eta^{\mu v}=\int_{k} \frac{\partial}{\partial k_{v}} \frac{k^{\mu}}{\left(k^{2}-m^{2}\right)^{2-w}}$,

$\left(\xi_{2 w}-v_{2 w}\right) \eta^{\{\mu \nu} \eta^{\alpha \beta\}}=\int_{k} \frac{\partial}{\partial k_{v}} \frac{2(2-w) k^{\mu} k^{\alpha} k^{\beta}}{\left(k^{2}-m^{2}\right)^{3-w}}$,

$$
\begin{aligned}
& \left(\sigma_{2 w}-\xi_{2 w}\right) \eta^{\{\mu v} \eta^{\alpha \beta} \eta^{\gamma \delta\}} \\
& =\int_{k} \frac{\partial}{\partial k_{v}} \frac{4(3-w)(2-w) k^{\mu} k^{\alpha} k^{\beta} k^{\gamma} k^{\delta}}{\left(k^{2}-m^{2}\right)^{4-w}}
\end{aligned}
$$

and

$$
\begin{aligned}
& \left(\omega_{2 w}-\sigma_{2 w}\right) \eta^{\{\mu \nu} \eta^{\alpha \beta} \eta^{\gamma \delta} \eta^{\epsilon \zeta\}} \\
& =\int_{k} \frac{\partial}{\partial k_{v}} \frac{8(4-w)(3-w)(2-w) k^{\mu} k^{\alpha} k^{\beta} k^{\gamma} k^{\delta} k^{\epsilon} k^{\zeta}}{\left(k^{2}-m^{2}\right)^{5-w}}
\end{aligned}
$$

We see that Eqs. (8)-(11) are undetermined because they are differences between divergent quantities. Each regularization scheme gives a different value for these terms. However, as physics should not depend on the schemes applied,

\footnotetext{
1 The Lorentz indices between brackets stand for permutations, i.e. $A^{\left\{\alpha_{1} \cdots \alpha_{n}\right\}} B^{\left\{\beta_{1} \cdots \beta_{n}\right\}}=A^{\alpha_{1} \cdots \alpha_{n}} B^{\beta_{1} \cdots \beta_{n}}+$ sum over permutations between the two sets of indices $\alpha_{1} \cdots \alpha_{n}$ and $\beta_{1} \cdots \beta_{n}$.
} 
we leave these terms to be arbitrary until the end of the calculation, fixing them by symmetry constraints or phenomenology, when it applies [8].

It is noteworthy that this prescription is not the usual one, since we do not evaluate divergent integrals or regularization dependent quantities neither do we introduce a regulator or further parameters usually introduced in explicit regularization procedures. We do assume the existence of a regulator in order to give sense to the manipulation (4). However, we do not say which one. That is because the introduction of an explicit regulator and additional parameters usually breaks symmetries of the theory and it makes the renormalization procedure more laborious.

Besides not modifying the theory, such as changing the dimension of space-time or breaking gauge or Lorentz symmetry spuriously, we can support or differ controversial results of the literature, which are most of the times caused by regularization dependent quantities like surface terms in (8)(11). If those terms remain in the finite part of the amplitude, it can be arbitrary and regularization dependent although of being finite. Therefore, we carry those terms till the end of the calculation and fix them using a symmetry requirement, the fulfillment of a Ward identity, for instance.

We should also emphasize that although IR was consistently built for multi-loop calculation in scalar field theories [44], its validity is questionable for arbitrary loop order in other theories and in curved space. If one works in a momentum space framework, one must ensure that causality and locality are guaranteed in all orders of perturbation theory. In differential regularization [45], for instance, it was shown that those principles hold at lower-order even in curved space [46]. However, we do not worry with that in the present case since we perform only one-loop calculations.

\section{One-loop correction to the graviton propagator and the trace anomaly}

We consider the semi-classical approach of gravitation where matter fields are quantized in a classical curved background (see [43] for a review). The action for scalar, fermion, and abelian vector are, respectively,

$$
\begin{aligned}
& S_{s}=\frac{1}{2} \int d^{4} x \sqrt{-g}\left(g^{\mu \nu} \partial_{\mu} \phi \partial_{\nu} \phi+\xi R \phi^{2}\right), \\
& S_{f}=i \int d^{4} x e e_{a}^{\mu} \bar{\psi} \gamma^{a} D_{\mu} \psi
\end{aligned}
$$

and

$$
S_{v}=-\frac{1}{4} \int d^{4} x \sqrt{-g} F^{\mu v} F_{\mu v}
$$

where $e_{a}^{\mu}$ is the tetrad, $e=\operatorname{det} e_{a}^{\mu}$, and $\xi$ is the non-minimal coupling.

In Eq. (17), in order to couple fermions with the gravitational field, we need to define the covariant derivative,

$D_{\mu} \psi=\partial_{\mu} \psi+\frac{1}{2} \omega_{\mu a b} \sigma^{a b} \psi$,

where $\omega_{\mu a b}$ is the spin connection, which depends on the tetrad, and $\sigma^{a b}=\frac{1}{4}\left[\gamma^{a}, \gamma^{b}\right]$, with $\gamma^{a}$ representing the Dirac matrices.

The actions expressed by (17) and (18) are classically conformal invariant and so is the action (16) in the conformal limit $\xi \rightarrow 1 / 6$. To compute the classical breaking we have first to calculate one-loop corrections to the graviton propagator. In order to do this we first consider the weak field approximation, i.e. we use the following expansions for the metric and the tetrad:

$g^{\mu \nu}=\eta^{\mu \nu}+\kappa h^{\mu \nu}$

and

$e_{\mu a}=\eta_{\mu a}+\frac{1}{2} \kappa h_{\mu a}$.

Thus, using Eqs. (20) and (21) in (16), (17), and (18), we obtain the Feynman rules up to first order in $\kappa$. We list them in Fig. 1.

Diagrams contributing to one-loop correction to the graviton propagator are presented in Fig. 2. The finite part responsible for the quantum breaking of conformal symmetry comes from diagrams (a), (b), and (c). Loop diagrams (d), (e), and (f) contribute only with quartic and quadratic divergences. Quadratic divergences for massless fields are made zero in dimensional regularization [47] and in implicit regularization [48]. Quartic divergences are unphysical in the sense that they do not contribute for physical quantities, like logarithmic divergences do when deriving the running of coupling constants, for instance. Both divergences also come from diagrams (a), (b), and (c). Using symmetric integration, like $k^{\mu} k^{\nu} \rightarrow \frac{1}{4} \eta^{\mu \nu} k^{2}$, all quartic divergences can be transformed in a single form $\int^{\Lambda} \frac{d^{4} k}{(2 \pi)^{4}}$, which can be subtracted by a suitable cosmological counter-term (see [49] for details).

Therefore, we have to calculate the following amplitudes:

$$
\begin{aligned}
\Pi_{(\mathrm{a})}^{\mu \nu \alpha \beta}(p)= & \frac{1}{2} \int_{k} V_{s}^{\mu \nu}(p, k, k+p) \frac{i}{k^{2}-m_{s}^{2}} \\
& \times V_{s}^{\alpha \beta}(p, k+p, k) \frac{i}{(k+p)^{2}-m_{s}^{2}},
\end{aligned}
$$




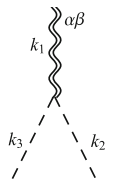

$$
\begin{aligned}
& V_{s}^{\alpha \beta}\left(k_{1}, k_{2}, k_{3}\right)=\frac{i \kappa}{2}\left[\eta^{\alpha \beta} k_{2} \cdot k_{3}-k_{2}^{\alpha} k_{3}^{\beta}-k_{3}^{\alpha} k_{2}^{\beta}-\right. \\
& \left.-2 \xi\left(k_{1}^{\alpha} k_{1}^{\beta}-k_{1}^{2} \eta^{\alpha \beta}\right)\right]
\end{aligned}
$$

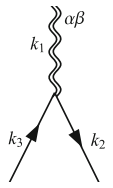

$$
\begin{aligned}
& V_{f}^{\alpha \beta}\left(k_{2}, k_{3}\right)=\frac{i \kappa}{8}\left[2 \eta^{\alpha \beta}\left(\not k_{2}+\not k_{3}\right)-\right. \\
& \left.-\gamma^{\alpha}\left(k_{2}+k_{3}\right)^{\beta}-\gamma^{\beta}\left(k_{2}+k_{3}\right)^{\alpha}\right]
\end{aligned}
$$

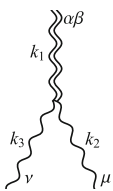

$V_{v}^{\alpha \beta \mu \nu}\left(k_{2}, k_{3}\right)=\frac{i \kappa}{2}\left[\left(\eta^{\mu\{\alpha} \eta^{\beta\} \nu}-\eta^{\mu \nu} \eta^{\alpha \beta}\right) k_{2} \cdot k_{3}+\left(\eta^{\alpha \beta} k_{2}^{\nu} k_{3}^{\mu}+\right.\right.$ $+\eta^{\mu \nu} k_{2}^{\alpha} k_{3}^{\beta}+\eta^{\mu \nu} k_{2}^{\beta} k_{3}^{\alpha}-\eta^{\alpha \nu} k_{2}^{\beta} k_{3}^{\mu}-\eta^{\mu \alpha} k_{2}^{\nu} k_{3}^{\beta}-\eta^{\mu \beta} k_{2}^{\nu} k_{3}^{\alpha}-$ $\left.\left.-\eta^{\beta \nu} k_{2}^{\alpha} k_{3}^{\mu}\right)\right]$

Fig. 1 Feynman rules for matter fields in linearized quantum gravity

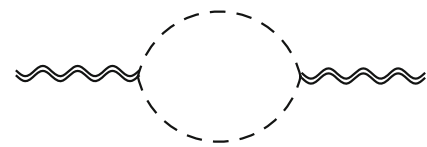

(a)

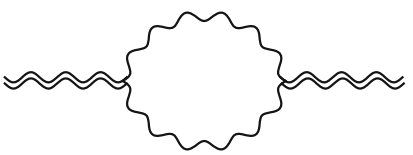

(b)

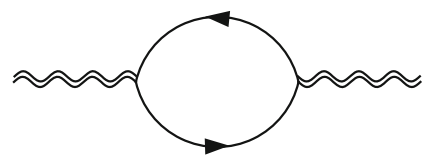

(c)

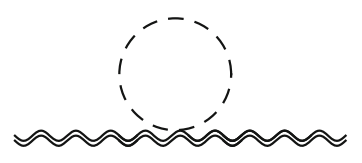

(d)

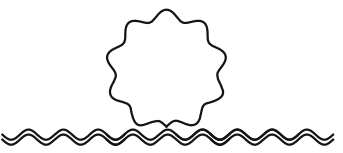

(e)

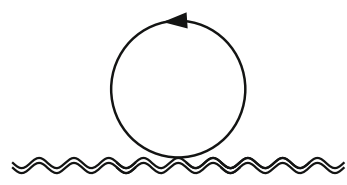

(f)
Fig. 2 One-loop corrections to the graviton propagator. The dashed, solid, waved, and double-waved lines stand for scalar, fermion, vector, and graviton, respectively

$$
\begin{aligned}
\Pi_{(\mathrm{b})}^{\mu \nu \alpha \beta}(p)= & \frac{1}{2} \int_{k} V_{v}^{\alpha \beta \lambda \theta}(k, k+p) \frac{-i \eta_{\lambda \gamma}}{k^{2}-m_{v}^{2}} \\
& \times V_{v}^{\mu \nu \gamma \delta}(k+p, k) \frac{-i \eta_{\delta \theta}}{(k+p)^{2}-m_{v}^{2}},
\end{aligned}
$$

$$
\begin{aligned}
\Pi_{(\mathrm{c})}^{\mu \nu \alpha \beta}(p)= & -\int_{k} \operatorname{Tr}\left[V_{f}^{\alpha \beta}(k, k+p) \frac{i}{\not k-m_{f}}\right. \\
& \left.\times V_{f}^{\mu \nu}(k+p, k) \frac{i}{\not k+\not p-m_{f}}\right] .
\end{aligned}
$$

In the equations above, $1 / 2$ is a symmetry factor and we have introduced fictitious masses in the propagators. This is necessary because, although the present integrals are infrared safe, expression (4) without mass will break the original integral in two infrared divergent parts. The limit $m_{i}^{2} \rightarrow 0$ is taken in the end. In this process a renormalization scale $\lambda \neq 0$ appears. Observe that the other part of the massive vector propagator in Eq. (23) does not contribute, since $k_{\lambda} k_{\gamma} V_{v}^{\alpha \beta \lambda \theta}(k, k+p)=0$ and $(k+p)_{\delta}(k+p)_{\theta} V_{v}^{\mu \nu \gamma \delta}(k+$ $p, k)=0$. For the sake of completeness, we list all regularized integrals coming from the expansion of Eqs. (22)-(24) in the appendix.

After taking the limits $m_{s} \rightarrow 0$ and $\xi \rightarrow \frac{1}{6}$, we find that amplitude (22) is transverse up to surface terms defined in Eqs. (8)-(11):

$$
\begin{aligned}
\frac{2}{\kappa^{2}} p_{\alpha} \Pi_{(\mathrm{a})}^{\mu \nu \alpha \beta}(p)= & \left(\frac{37}{48} p^{\beta} \eta^{\mu \nu} p^{2}+\frac{1}{16} p^{\mu} \eta^{\beta v} p^{2}\right. \\
& \left.+\frac{1}{16} p^{v} \eta^{\beta \mu} p^{2}+\frac{2}{3} p^{\mu} p^{\beta} p^{v}\right) p^{2} v_{0} \\
& -\left(\frac{29}{48} p^{\beta} \eta^{\mu v} p^{2}+\frac{1}{16} p^{\mu} \eta^{\beta v} p^{2}\right. \\
& \left.+\frac{1}{16} p^{v} \eta^{\beta \mu} p^{2}+\frac{1}{3} p^{\mu} p^{\beta} p^{v}\right) p^{2} \xi_{0} \\
& +\left(\frac{109}{8} p^{\beta} \eta^{\mu \nu} p^{2}+\frac{37}{8} p^{\mu} \eta^{\beta v} p^{2}\right. \\
& \left.+\frac{37}{8} p^{v} \eta^{\beta \mu} p^{2}+\frac{121}{4} p^{\mu} p^{\beta} p^{v}\right) p^{2} \sigma_{0} \\
& -\left(\frac{1}{8} p^{\beta} \eta^{\mu \nu} p^{2}+\frac{1}{8} p^{\mu} \eta^{\beta v} p^{2}\right. \\
& \left.+\frac{1}{8} p^{v} \eta^{\beta \mu} p^{2}+\frac{1}{2} p^{\mu} p^{\beta} p^{v}\right) p^{2} \omega_{0} .
\end{aligned}
$$

We see in Eq. (25) that gauge invariance, i.e. $p_{\alpha} \Pi_{a}^{\mu \nu \alpha \beta}(p)$ $=0$, holds if all indeterminacies expressed by surface terms are set to zero. That is not the only possible solution. There may exist relations between these surface terms which also would entail gauge invariance. In this case the finite result would be arbitrary [8,50-52]. We are going to discuss this in Sect. 6. The ambiguity in the $a^{\prime}$ term may be due to surface terms because they are often the source of ambiguities as we found in other models $[50,51,53]$. 
Our final result for the amplitude (22) is

$$
\begin{aligned}
\frac{2}{\kappa^{2}} & \Pi_{(\mathrm{a})}^{\mu \nu \alpha \beta}(p) \\
= & p^{2}\left(\eta^{\alpha \nu} \eta^{\beta \mu} p^{2}+\eta^{\alpha \mu} \eta^{\beta v} p^{2}-p^{\alpha} p^{\mu} \eta^{\beta \nu}\right. \\
& \left.-p^{\beta} p^{\mu} \eta^{\alpha \nu}-p^{\alpha} p^{v} \eta^{\beta \mu}-p^{\beta} p^{v} \eta^{\alpha \mu}\right) \\
& \times\left[\frac{23}{1800} b+\frac{1}{240}\left(I_{\log }\left(\lambda^{2}\right)-b \ln \left(-\frac{p^{2}}{\lambda^{2}}\right)\right)\right] \\
- & p^{2}\left(\eta^{\alpha \beta} \eta^{\mu v} p^{2}-p^{\alpha} p^{\beta} \eta^{\mu \nu}-p^{\mu} p^{v} \eta^{\alpha \beta}\right) \\
\times & {\left[\frac{7}{675} b+\frac{1}{360}\left(I_{\log }\left(\lambda^{2}\right)-b \ln \left(-\frac{p^{2}}{\lambda^{2}}\right)\right)\right] } \\
+ & \frac{1}{180}\left(\frac{41}{15} b+I_{\log }\left(\lambda^{2}\right)\right. \\
& \left.-b \ln \left(-\frac{p^{2}}{\lambda^{2}}\right)\right) p^{\alpha} p^{\beta} p^{\mu} p^{v} .
\end{aligned}
$$

This result agrees with [55] after identifying $I_{\log }\left(\lambda^{2}\right)$ as the divergent part. We now return to the curvature tensor from weak field approximation. In order to do this we write the corresponding covariant expression and we focus on the $\square R$ term

$$
\begin{aligned}
S & =\int d^{4} x \sqrt{-g}\left(\alpha_{1} C^{2}+\alpha_{2} R^{2}\right) \\
& \rightarrow \int d^{4} x \sqrt{-g}\left(2 \alpha_{1} W+\alpha_{2} R^{2}\right),
\end{aligned}
$$

where we replace $C^{2} \rightarrow 2 W=2 R_{\mu \nu}^{2}-\frac{2}{3} R^{2}$ since the Gauss-Bonnet topological invariant does not contribute to the propagator since it reduces in a topological surface term.

Applying the definition of the energy-momentum tensor for the action (27), we see that its trace is given by

$\left\langle T_{\mu}^{\mu}\right\rangle=\frac{-2}{\sqrt{-g}} g^{\mu \nu} \frac{\delta S}{\delta g^{\mu \nu}}=12 \alpha_{2} \square R$.

Therefore, all we have to do is to determine the constant $\alpha_{2}$. For this purpose, we write $W$ and $R^{2}$ in the weak field limit up to second order in $\kappa$ :

$$
\begin{aligned}
\int d^{4} x \sqrt{-g} R^{2}= & \int d^{4} x h^{\mu \nu}\left[\partial_{\mu} \partial_{\nu} \partial_{\alpha} \partial_{\beta}+\eta_{\mu \nu} \eta_{\alpha \beta} \partial^{4}\right. \\
& \left.-\left(\eta_{\mu \nu} \partial_{\alpha} \partial_{\beta} \partial^{2}+\eta_{\alpha \beta} \partial_{\mu} \partial_{\nu} \partial^{2}\right)\right] h^{\alpha \beta} \\
\int d^{4} x \sqrt{-g} W= & \int d^{4} x h^{\mu \nu}\left[\frac{1}{6} \partial_{\mu} \partial_{\nu} \partial_{\alpha} \partial_{\beta}-\frac{1}{12} \eta_{\mu \nu} \eta_{\alpha \beta} \partial^{4}\right. \\
& +\frac{1}{12}\left(\eta_{\mu \nu} \partial_{\alpha} \partial_{\beta} \partial^{2}+\eta_{\alpha \beta} \partial_{\mu} \partial_{\nu} \partial^{2}\right) \\
& +\frac{1}{8}\left(\eta_{\mu \alpha} \eta_{\nu \beta}+\eta_{\nu \alpha} \eta_{\mu \beta}\right) \partial^{4} \\
& \left.-\frac{1}{8} \eta_{\mu \alpha} \partial_{\nu} \partial_{\beta} \partial^{2}\right] h^{\alpha \beta}
\end{aligned}
$$

Replacing Eqs. (29) and (30) in Eq. (27) and comparing with Eq. (26) written in the position space (the action for the graviton propagator is $S=-\frac{1}{2} \int d^{4} x h^{\mu \nu} \bar{\Pi}_{\mu \nu \alpha \beta} h^{\alpha \beta}$, where $\bar{\Pi}_{\mu \nu \alpha \beta}$ is the Fourier transform of Eq. (26)), we get $12 \alpha_{2}=$ $\frac{1}{180(4 \pi)^{2}}$. Therefore, our result for the anomaly is

$\left\langle T_{\mu}^{\mu}\right\rangle_{\text {scalar }}=\frac{1}{180(4 \pi)^{2}} \square R$

This result agrees with the one obtained in [16,26-28,55], where it was applied dimensional regularization, $\zeta$-function regularization, point-splitting regularization and proper time cut-off regularization, respectively. We proceed using the same idea to obtain the anomaly contributions coming from vector and spinor fields. The result of the one-loop correction to the graviton propagator for the amplitudes (23) and (24) are, respectively (if we set again the surface terms to zero, gauge invariance holds, i.e. $p_{\alpha} \Pi_{(\mathrm{b})}^{\mu \nu \alpha \beta}(p)=0$ and $\left.p_{\alpha} \Pi_{(\mathrm{c})}^{\mu \nu \alpha \beta}(p)=0\right)$,

$$
\begin{aligned}
\frac{2}{\kappa^{2}} \Pi_{(\mathrm{b})}^{\mu \nu \alpha \beta}(p)= & p^{2}\left(\eta^{\alpha \nu} \eta^{\beta \mu} p^{2}+\eta^{\alpha \mu} \eta^{\beta \nu} p^{2}-p^{\alpha} p^{\mu} \eta^{\beta v}\right. \\
& \left.-p^{\beta} p^{\mu} \eta^{\alpha \nu}-p^{\alpha} p^{\nu} \eta^{\beta \mu}-p^{\beta} p^{\nu} \eta^{\alpha \mu}\right) \\
& \times\left[\frac{4}{75} b+\frac{1}{20}\left(I_{\log }\left(\lambda^{2}\right)-b \ln \left(-\frac{p^{2}}{\lambda^{2}}\right)\right)\right] \\
& -p^{2}\left(\eta^{\alpha \beta} \eta^{\mu \nu} p^{2}-p^{\alpha} p^{\beta} \eta^{\mu \nu}-p^{\mu} p^{v} \eta^{\alpha \beta}\right) \\
& \times\left[\frac{1}{450} b+\frac{1}{30}\left(I_{\log }\left(\lambda^{2}\right)-b \ln \left(-\frac{p^{2}}{\lambda^{2}}\right)\right)\right] \\
& +\frac{1}{15}\left(\frac{47}{30} b+I_{\log }\left(\lambda^{2}\right)-b \ln \left(-\frac{p^{2}}{\lambda^{2}}\right)\right) \\
& \times p^{\alpha} p^{\beta} p^{\mu} p^{v}
\end{aligned}
$$

and

$$
\begin{aligned}
\frac{2}{\kappa^{2}} \Pi_{(\mathrm{c})}^{\mu \nu \alpha \beta}(p)= & p^{2}\left(\eta^{\alpha \nu} \eta^{\beta \mu} p^{2}+\eta^{\alpha \mu} \eta^{\beta \nu} p^{2}-p^{\alpha} p^{\mu} \eta^{\beta \nu}\right. \\
& \left.-p^{\beta} p^{\mu} \eta^{\alpha \nu}-p^{\alpha} p^{v} \eta^{\beta \mu}-p^{\beta} p^{v} \eta^{\alpha \mu}\right) \\
& \times\left[\frac{3}{50} b+\frac{1}{40}\left(I_{\log }\left(\lambda^{2}\right)-b \ln \left(-\frac{p^{2}}{\lambda^{2}}\right)\right)\right] \\
& -p^{2}\left(\eta^{\alpha \beta} \eta^{\mu \nu} p^{2}-p^{\alpha} p^{\beta} \eta^{\mu \nu}-p^{\mu} p^{v} \eta^{\alpha \beta}\right) \\
& \times\left[\frac{23}{450} b+\frac{1}{60}\left(I_{\log }\left(\lambda^{2}\right)-b \ln \left(-\frac{p^{2}}{\lambda^{2}}\right)\right)\right] \\
& +\frac{1}{30}\left(\frac{31}{15} b+I_{\log }\left(\lambda^{2}\right)-b \ln \left(-\frac{p^{2}}{\lambda^{2}}\right)\right) \\
& \times p^{\alpha} p^{\beta} p^{\mu} p^{v} .
\end{aligned}
$$

The corresponding values for the constant $\alpha_{2}$ for Eqs. (32) and (33) are $12 \alpha_{2}=-\frac{1}{10(4 \pi)^{2}}$ and $12 \alpha_{2}=\frac{1}{30(4 \pi)^{2}}$, respectively. Multiplying each diagram by the number of fields, our final result is 


$$
\begin{aligned}
\left\langle T_{\mu}^{\mu}\right\rangle & =\left(\frac{1}{180(4 \pi)^{2}} N_{s}+\frac{1}{30(4 \pi)^{2}} N_{f}-\frac{1}{10(4 \pi)^{2}} N_{v}\right) \square R \\
& =\beta_{3} \square R .
\end{aligned}
$$

Therefore, we found that apparently no ambiguity appears in the massless case and if we require gauge symmetry that fixes the surface terms to zero. This result agrees with all regularization methods [26-30] but the one obtained by dimensional regularization $[14,16]$. This may suggest the result (34) is universal and dimensional regularization provides a different result because of a hard breaking of conformal symmetry. However, as we are going to see in Sect. 5, there is an inherent ambiguity associated with the renormalization. Moreover, in Sect. 6 we present how this anomaly can be plagued by the arbitrary surface term, which also makes its result ambiguous.

\section{Renormalization}

We now perform the one-loop renormalization. Therefore, we write the one-loop renormalized action corresponding to the calculation of the previous section

$S_{R}\left(a_{1}, a_{2}\right)=S_{\text {vacuum }}\left(a_{1}^{(0)}, a_{2}^{(0)}\right)+\bar{\Gamma}^{(1)}+\Delta S_{\text {vacuum }}$,

where $S_{\text {vacuum }}\left(a_{1}^{(0)}, a_{2}^{(0)}\right)=\int d^{4} x \sqrt{-g}\left(a_{1}^{(0)} C^{2}+a_{2}^{(0)} R^{2}\right)$ is the vacuum action, $\bar{\Gamma}^{(1)}$ is the one-loop effective action and $\Delta S_{\text {vacuum }}$ is the counter-term action.

In order to renormalize, we seize the results of Sect. 4. Considering, for instance, the photon correction given by Eq. (32), we have the following effective action:

$$
\begin{aligned}
\bar{\Gamma}^{(1)}= & \frac{1}{(4 \pi)^{2}} \int d^{4} x \sqrt{-g}\left[C _ { \mu \nu \alpha \beta } \left(-\frac{4}{75}\right.\right. \\
& \left.+\frac{1}{20}\left((4 \pi)^{2} i I_{\log }\left(\lambda^{2}\right)+\ln \left(\frac{\square}{\lambda^{2}}\right)\right)\right) C^{\mu \nu \alpha \beta} \\
& \left.-\frac{1}{120} R^{2}\right] .
\end{aligned}
$$

We may choose $\Delta S_{\text {vacuum }}=-\frac{i}{20} \int d^{4} x \sqrt{-g} I_{\log }\left(\lambda^{2}\right) C^{2}$. We add this counter-term in order to remove the divergent integral $I_{\log }\left(\lambda^{2}\right)$. This is equivalent to the $M S$ renormalization scheme as we have shown in Ref. [54]. However, it is also possible to add a finite local counter-term of the form $\frac{1}{(4 \pi)^{2}} \alpha \int d^{4} x \sqrt{-g} R^{2}$ since it is a vacuum term and it does not break conformal symmetry of the quantum fields. Considering these counter-terms, we end up with the following renormalized action:

$$
\begin{aligned}
S_{R}\left(a_{1}, a_{2}\right)= & \frac{1}{(4 \pi)^{2}} \int d^{4} x \sqrt{-g} \\
& \times\left[C_{\mu \nu \alpha \beta}\left(a_{1}^{(0)}-\frac{4}{75}+\frac{1}{20} \ln \left(\frac{\square}{\lambda^{2}}\right)\right) C^{\mu \nu \alpha \beta}\right.
\end{aligned}
$$

$$
\begin{aligned}
& \left.+\left(a_{2}^{(0)}-\frac{1}{120}+\alpha\right) R^{2}\right] \\
= & \int d^{4} x \sqrt{-g}\left(a_{1} C^{2}+a_{2} R^{2}\right) .
\end{aligned}
$$

Requiring that Eq. (37) must not depend on the renormalization group scale $\lambda$, we find the one-loop $\beta$-function

$\beta_{1}=\lambda \frac{\partial a_{1}}{\partial \lambda}=2 \lambda^{2} \frac{\partial a_{1}}{\partial \lambda^{2}}=-\frac{1}{10(4 \pi)^{2}}$.

Following the same idea, the contributions coming from the scalar and the spinor field are $\beta_{1}=-\frac{1}{120(4 \pi)^{2}}$ and $\beta_{1}=-\frac{1}{20(4 \pi)^{2}}$, respectively. This result agrees with $[55,56]$ where it was applied the $\overline{M S}$ scheme. In this case we have found only the ultraviolet behavior of the $\beta$-function since we consider massless matter fields in a curved background.

Clearly, the addition of the local finite counter-term generates an arbitrariness in the conformal anomaly. Applying Eq. (28) for the action (37) we find the arbitrary result

$$
\left\langle T_{\mu}^{\mu}\right\rangle_{\text {vector }}=\frac{1}{(4 \pi)^{2}}\left(-\frac{1}{10}+12 \alpha\right) \square R
$$

The result of Eq. (39) is compatible with regularization schemes which breaks hardly conformal symmetry such as dimensional regularization, as mentioned before. The result also agrees with the obtained in Pauli-Villars regularization, where an ambiguous result can also be found for the massive theory $[42,56]$. In the next section, we show that an arbitrariness also appears if we do not set all surfaces terms to zero.

\section{Arbitrariness in the conformal anomaly}

We return to the previous amplitudes in order to see what happens if we do not set all surfaces terms to zero. For instance, consider again the amplitude (23). However, this time we investigate if there is a relation between surface terms which also make the final amplitude gauge invariant. As before we use the gauge Ward identity in order to fix those arbitrary surface terms. After taking the limit $m \rightarrow 0$, we find that amplitude (23) is transverse up to surfaces terms

$$
\begin{aligned}
\frac{2}{\kappa^{2}} p_{\alpha} \Pi_{(\mathrm{b})}^{\mu \nu \alpha \beta}(p)= & \left(\frac{1}{8} p^{\beta} \eta^{\mu v} p^{2}+p^{\mu} \eta^{\beta \nu} p^{2}\right. \\
& \left.+p^{v} \eta^{\beta \mu} p^{2}+p^{\mu} p^{\beta} p^{\nu}\right) \\
& \times p^{2} v_{0}-\left(\frac{1}{8} p^{\beta} \eta^{\mu v} p^{2}+\frac{3}{4} p^{\mu} \eta^{\beta \nu} p^{2}\right.
\end{aligned}
$$




$$
\begin{aligned}
& \left.+\frac{3}{4} p^{v} \eta^{\beta \mu} p^{2}+\frac{1}{2} p^{\mu} p^{\beta} p^{v}\right) p^{2} \xi_{0} \\
& +\left(\frac{37}{4} p^{\beta} \eta^{\mu \nu} p^{2}+\frac{73}{4} p^{\mu} \eta^{\beta v} p^{2}\right. \\
& \left.+\frac{73}{4} p^{v} \eta^{\beta \mu} p^{2}+\frac{121}{2} p^{\mu} p^{\beta} p^{v}\right) p^{2} \sigma_{0} \\
& -\left(\frac{1}{4} p^{\beta} \eta^{\mu \nu} p^{2}+\frac{1}{4} p^{\mu} \eta^{\beta v} p^{2}\right. \\
& \left.+\frac{1}{4} p^{v} \eta^{\beta \mu} p^{2}+p^{\mu} p^{\beta} p^{v}\right) p^{2} \omega_{0}=0
\end{aligned}
$$

As in Eq. (25), setting all surface terms to zero is a possible solution. However, we can easily see that it is possible to establish a relation between them which would also satisfy (40). Considering the tensorial structure, we see that requiring gauge invariance gives us the relations

$$
\begin{aligned}
v_{0}-\xi_{0}+74 \sigma_{0}-2 \omega_{0} & =0, \\
4 v_{0}-3 \xi_{0}+73 \sigma_{0}-\omega_{0} & =0, \\
2 v_{0}-\xi_{0}+121 \sigma_{0}-2 \omega_{0} & =0 .
\end{aligned}
$$

Since the parameters are overdetermined by equations above we may write $v_{0}=-47 \sigma_{0}, \xi_{0}=-\frac{257}{5} \sigma_{0}$, and $\omega_{0}=\frac{196}{5} \sigma_{0}$. This means that gauge invariance was not sufficient to fix all the arbitrary terms. Consequently, we can replace $v_{0}, \xi_{0}$, and $\omega_{0}$ in the amplitude and the final answer now depends on the arbitrary surface term $\sigma_{0}$. As a result, the anomaly become arbitrary because it depends on the arbitrary surface term

$$
\begin{aligned}
\left\langle T_{\mu}^{\mu}\right\rangle_{\text {vector }} & =-\frac{1}{(4 \pi)^{2}}\left(\frac{1}{10}+\frac{497}{15} \sigma_{0}\right) \square R \\
& =\frac{1}{(4 \pi)^{2}}\left(-\frac{1}{10}+\sigma_{0}^{\prime}\right) \square R
\end{aligned}
$$

This result is compatible with the arbitrariness that appears in renormalization, as presented in the previous section. It also agrees with the result found in dimensional regularization of Ref. [14] and in Pauli-Villars regularization [14,42,56].

In order to support our result, we also calculated the anomaly for the massive case. In this case, we found the same ambiguity as appeared in (44) (massless case), according to $[14,42,56]$. Although in the latter case the ambiguity was found only in the massive theory, our result shows that the ambiguity in the conformal anomaly appears even in the massless case.

\section{Conclusion}

In this paper, we considered an implicit momentum space regularization derivation of the one-loop conformal anomaly in order to shed some light on controversies raised in the literature in which some finite breaking terms are ambiguous. Our approach is specially tailored to study quantum symmetry breakings. In this approach, regularization dependent indeterminacies expressed by surface terms are identified to be fixed on symmetry grounds. However, as in the present case the symmetry content of the theory is not sufficient to fix all the arbitrary terms and the finite part of the amplitude is ambiguous and regularization dependent although of being finite. As a result, we find that there is an unavoidable arbitrariness in the conformal anomaly even in the massless case. Our result is equivalent to the usual subtraction procedure of including an $\int d^{4} x \sqrt{-g} R^{2}$-term in the renormalized action.

Acknowledgments The authors are grateful to Ilya Shapiro for clarifying discussions. A. R. V. acknowledges financial support by CNPq. M. S. acknowledges research grants from $\mathrm{CNPq}$ and Durham University for the kind hospitality. J. C. C. F. acknowledges financial support by CAPES. G. G. acknowledges financial support by FAPEMIG. This work is dedicated to the memory of professor M. C. Nemes.

Open Access This article is distributed under the terms of the Creative Commons Attribution 4.0 International License (http://creativecomm ons.org/licenses/by/4.0/), which permits unrestricted use, distribution, and reproduction in any medium, provided you give appropriate credit to the original author(s) and the source, provide a link to the Creative Commons license, and indicate if changes were made.

Funded by SCOAP ${ }^{3}$.

\section{Appendix}

The results of the regularized integrals in the massless limit are

$$
\begin{aligned}
& \int_{k} \frac{1}{k^{2}(k+p)^{2}}=I_{\log }\left(\lambda^{2}\right)+2 b-b \ln \left(-\frac{p^{2}}{\lambda^{2}}\right), \\
& \int_{k} \frac{k^{2}}{k^{2}(k+p)^{2}}=-p^{2} v_{0}, \\
& \int_{k} \frac{k^{2} k^{\alpha}}{k^{2}(k+p)^{2}}=p^{2} p^{\alpha}\left(\xi_{0}-v_{0}\right), \\
& \int_{k} \frac{k^{4}}{k^{2}(k+p)^{2}}=p^{4}\left(3 v_{0}-2 \xi_{0}\right), \\
& \int_{k} \frac{k^{\alpha}}{k^{2}(k+p)^{2}}=\frac{1}{2} p^{\alpha}\left[-I_{\log }\left(\lambda^{2}\right)+v_{0}-2 b+b \ln \left(-\frac{p^{2}}{\lambda^{2}}\right)\right], \\
& \int_{k} \frac{k^{\alpha} k^{\beta}}{k^{2}(k+p)^{2}}=\left(\frac{1}{3} p^{\alpha} p^{\beta}-\frac{1}{12} p^{2} \eta^{\alpha \beta}\right)
\end{aligned}
$$




$$
\begin{aligned}
& \times\left[I_{\log }\left(\lambda^{2}\right)-b \ln \left(-\frac{p^{2}}{\lambda^{2}}\right)\right] \\
& -\left(\frac{1}{3} p^{\alpha} p^{\beta}+\frac{1}{6} p^{2} \eta^{\alpha \beta}\right) \xi_{0} \\
& +\frac{1}{4} p^{2} \eta^{\alpha \beta} v_{0}+\frac{13}{18} b p^{\alpha} p^{\beta}-\frac{2}{9} p^{2} b \eta^{\alpha \beta},
\end{aligned}
$$

$$
\begin{aligned}
\int_{k} \frac{k^{\mu} k^{\alpha} k^{\beta}}{k^{2}(k+p)^{2}}= & \frac{1}{24}\left(p^{\{\mu} \eta^{\alpha \beta\}} p^{2}-6 p^{\alpha} p^{\beta} p^{\mu}\right) \\
& \times\left[I_{\log }\left(\lambda^{2}\right)-\xi_{0}-b \ln \left(-\frac{p^{2}}{\lambda^{2}}\right)\right] \\
& +3\left(p^{\{\mu} \eta^{\alpha \beta\}} p^{2}+2 p^{\alpha} p^{\beta} p^{\mu}\right) \sigma_{0} \\
& +\frac{1}{9} b p^{\{\mu} \eta^{\alpha \beta\}} p^{2}-\frac{7}{12} b p^{\mu} p^{\alpha} p^{\beta}
\end{aligned}
$$

$$
\begin{aligned}
\int_{k} \frac{k^{2} k^{\alpha} k^{\beta}}{k^{2}(k+p)^{2}}= & \frac{1}{4} p^{4} \eta^{\alpha \beta}\left(\xi_{0}-v_{0}\right) \\
& -6 p^{2}\left(4 p^{\alpha} p^{\alpha}+\eta^{\alpha \beta} p^{2}\right) \sigma_{0},
\end{aligned}
$$

$$
\begin{aligned}
\int_{k} \frac{k^{\mu} k^{v} k^{\alpha} k^{\beta}}{k^{2}(k+p)^{2}} \\
=\frac{1}{240}\left(\eta^{\{\mu \nu} \eta^{\alpha \beta\}} p^{4}-3 p^{2} p^{\{\mu} p^{v} \eta^{\alpha \beta\}}\right. \\
\left.+48 p^{\alpha} p^{\beta} p^{\mu} p^{v}\right)\left[I_{\log }\left(\lambda^{2}\right)-b \ln \left(-\frac{p^{2}}{\lambda^{2}}\right)\right] \\
+\frac{1}{48} \eta^{\{\mu \nu} \eta^{\alpha \beta\}} p^{4}\left(26 \sigma_{0}-\xi_{0}-\frac{6}{5} \omega_{0}\right) \\
+\frac{1}{48} p^{\{\alpha} p^{\beta} \eta^{\mu \nu\}} p^{2}\left(26 \sigma_{0}+\xi_{0}-\frac{12}{5} \omega_{0}\right) \\
+\frac{1}{600} b\left(\frac{23}{3} p^{4} \eta^{\{\alpha \beta} \eta^{\mu \nu\}}-\frac{41}{2} p^{2} p^{\{\alpha} p^{\beta} \eta^{\mu \nu\}}\right) \\
+\frac{149}{300} b p^{\mu} p^{\nu} p^{\alpha} p^{\beta}-\frac{1}{5} p^{\mu} p^{\nu} p^{\alpha} p^{\beta} \omega_{0},
\end{aligned}
$$

where $\lambda$ is the renormalization group scale and $b \equiv \frac{i}{(4 \pi)^{2}}$. For the sake of simplicity we omit quartic divergent integrals. The surface terms are defined in Sect. 3.

\section{References}

1. I.L. Buchbinder, S.D. Odintsov, I.L. Shapiro, Effective Action in Quantum Gravity (Institute of Physics Publishing, Bristol, 1992)

2. J. Maldacena, Int. J. Theor. Phys. 38, 1113 (1999)

3. M. Natsuume. arXiv:hep-th/14093575

4. J.S. Bell, R. Jackiw, Nuovo Cimento 60, 47 (1969)

5. S.L. Adler, Phys. Rev. 177, 2426 (1969)

6. D.M. Capper, M.J. Duff, L. Halpern, Phys. Rev. D 10, 461 (1974)

7. L.S. Brown, Phys. Rev. D 15, 1469 (1977)

8. R. Jackiw, Int. J. Mod. Phys. B 14, 2011 (2000)

9. L.C. Ferreira, A.L. Cherchiglia, B. Hiller, M. Sampaio, M.C. Nemes, Phys. Rev. D 86, 025016 (2012)

10. G. 't Hooft, M. Veltman, Nucl. Phys. B 44, 189 (1972)
11. O.A. Battistel, A.L. Mota, M.C. Nemes, Mod. Phys. Lett. A 13, 1597 (1998)

12. L.A.M. Souza, M. Sampaio, M.C. Nemes, Phys. Lett. B 632, 717 (2006)

13. M.J. Duff, Class. Quantum Grav. 11, 1387 (1994)

14. M. Asorey, E.V. Gorbar, I.L. Shapiro, Class. Quantum Grav. 21, 163 (2004)

15. D.M. Capper, M.J. Duff, Nucl. Phys. B 82, 147 (1974)

16. D.M. Capper, M.J. Duff, Nuovo Cimento A 23, 173 (1974)

17. D.M. Capper, M.J. Duff, Phys. Lett. A 53, 361 (1975)

18. R. Kallosh, Phys. Lett. B 55, 321 (1974)

19. E.S. Fradkin, G.A. Vilkovisky, Phys. Lett. B 73, 209 (1978)

20. S.L. Adler, J. Lieberman, Y.J. Ng, Ann. Phys. 106, 279 (1977)

21. F. Englert, C. Truffin, R. Gastmans, Nucl. Phys. B 117, 407 (1976)

22. I. Antoniadis, N.C. Tsamis, Phys. Lett. B 144, 55 (1984)

23. I. Antoniadis, J. Iliopoulus, T.N. Tomaras, Nucl. Phys. B 261, 157 (1985)

24. I. Antoniadis, C. Kounnas, D.V. Nanopoulos, Phys. Lett. B 162, 309 (1985)

25. C.G. Bollini, J.J. Giambiagi, Nuovo Cimento B 12, 20 (1972)

26. S. Hawking, Commun. Math. Phys 55, 133 (1977)

27. S.M. Christensen, Phys. Rev. D 14, 2490 (1976)

28. S.M. Christensen, Phys. Rev. D 17, 946 (1978)

29. A.O. Barvinsky, G.A. Vilkovisky, Nucl. Phys. B 333, 471 (1990)

30. A.O. Barvinsky, Y.V. Gusev, G.A. Vilkovisky, V.V. Zhitnikov, Nucl. Phys. B 439, 561 (1995)

31. M. Henningson, K. Skenderis, J. High Energy Phys. JHEP07 (1998) 023

32. N. Boulanger, J. High Energy Phys. JHEP07 (2007) 069

33. N. Boulanger, Phys. Rev. Lett. 98, 261302 (2007)

34. S.M. Christensen, S.A. Fulling, Phys. Rev. D 15, 2088 (1977)

35. R. Balbinot, A. Fabbri, I. Shapiro, Nucl. Phys. B 559, 301 (1999)

36. R. Balbinot, A. Fabbri, I.L. Shapiro, Phys. Rev. Lett. 83, 1494 (1999)

37. A.A. Starobinski, Phys. Lett. B 91, 99 (1980)

38. I.L. Shapiro, Int. J. Mod. Phys 11, 1159 (2002)

39. S. Nojiri, S.D. Odintsov, Phys. Rev. D 59, 044026 (1999)

40. I.H. Brevik, S.D. Odintsov, Phys. Lett. B 475, 247 (2000)

41. S. Nojiri, S.D. Odintsov, Phys. Lett. B 484, 119 (2000)

42. M. Asorey, G. Berredo-Peixoto, I.L. Shapiro, Phys. Rev. D 74, 124011 (2006)

43. I.L. Shapiro, Class. Quantum Grav. 25, 103001 (2008)

44. A.L. Cherchiglia, M. Sampaio, M.C. Nemes, Int. J. Mod. Phys. A 26, 2591 (2011)

45. D.Z. Freedman, K. Johnson, J.I. Latorre, Nucl. Phys. B 371, 353 (1999)

46. D. Prange, J. Phys. A Math. Gen. 32, 2225 (1999)

47. G. Leibbrandt, Rev. Mod. Phys. 47, 849 (1975)

48. A.L. Cherchiglia, A.R. Vieira, B. Hiller, M. Sampaio, A.P. Baêta Scarpelli, Ann. Phys. 351, 751 (2014)

49. R. Utiyama, B.S. DeWitt, J. Math. Phys. 3, 608 (1962)

50. A.P. Baêta Scarpelli, M. Sampaio, M.C. Nemes, B. Hiller, Eur. Phys. J. C 56, 571 (2008)

51. J.C.C. Felipe, A.R. Vieira, A.L. Cherchiglia, A.P. Baêta Scarpelli, M. Sampaio, Phys. Rev. D 89, 105034 (2014)

52. G. Gazzola, H.G. Fargnoli, A.P. Baêta Scarpelli, M. Sampaio, M.C. Nemes, J. Phys. G 39, 035002 (2012)

53. A.L. Cherchiglia, L.A. Cabral, M.C. Nemes, M. Sampaio, Phys. Rev. D 87, 065011 (2013)

54. M. Sampaio, A.P. Baêta Scarpelli, B. Hiller, A. Brizola, M.C. Nemes, S. Gobira, Phys. Rev. D 65, 125023 (2002)

55. E.V. Gorbar, I.L. Shapiro, J. High Energy Phys. 02, 021 (2003)

56. E.V. Gorbar, I.L. Shapiro, J. High Energy Phys. 06, 004 (2003) 